\title{
A Fréchet Mean Approach for Compressive Sensing Date Acquisition and Reconstruction in Wireless Sensor Networks
}

\author{
Wei Chen, Student Member, IEEE, Miguel R. D. Rodrigues, Member, IEEE, and Ian J. Wassell
}

\begin{abstract}
Compressive sensing leverages the compressibility of natural signals to trade off the convenience of data acquisition against computational complexity of data reconstruction. Thus, CS appears to be an excellent technique for data acquisition and reconstruction in a wireless sensor network (WSN) which typically employs a smart fusion center (FC) with a high computational capability and several dumb front-end sensors having limited energy storage. This paper presents a novel signal reconstruction method based on CS principles for applications in WSNs. The proposed method exploits both the intra-sensor and inter-sensor correlation to reduce the number of samples required for reconstruction of the original signals. The novelty of the method relates to the use of the Fréchet mean of the signals as an estimate of their sparse representations in some basis. This crude estimate of the sparse representation is then utilized in an enhanced data recovering convex algorithm, i.e., the penalized $\ell_{1}$ minimization, and an enhanced data recovering greedy algorithm, i.e., the precognition matching pursuit (PMP). The superior reconstruction quality of the proposed method is demonstrated by using data gathered by a WSN located in the Intel Berkeley Research lab.
\end{abstract}

\section{INTRODUCTION}

W IRELESS sensor networks (WSN) provide a flexible way to monitor the physical parameters of the environment through the deployment of a large number of sensor nodes (SN). There are three important challenges in WSNs, specifically network lifetime, computational capability and bandwidth constraints. The first two challenges result from the size and cost limitation of SNs, while the third challenge is due to the physical character of the wireless channel.

Compressive sensing (CS) is a new sampling paradigm, which leverages the compressibility of signals to reduce the number of samples required for reconstruction and have been applied to a wide range of applications [1]-[3]. Using the CS technique as the data acquisition approach in WSNs can significantly reduce the energy consumed in the process of sampling and transmission through the network, and also lower the wireless bandwidth requirements for communication.

The traditional approach to measure environmental information in WSNs, e.g., temperature, humidity, light and sound, is

The work of M. R. D. Rodrigues was supported by the Fundação para a Ciência e a Tecnologia through the research project CMU-PT/SIA/0026/2009.

W. Chen and I. J. Wassell are with the Digital Technology Group, Computer Laboratory, University of Cambridge, Cambridge, U.K. (e-mail: wc253, ijw24@cam.ac.uk).

M. R. D. Rodrigues was with Instituto de Telecomunicações, Departamento de Ciência de Computadores, Universidade do Porto, Porto, Portugal. He is now with the Dept. E\&EE, University College London, London, U.K. (e-mail: m.rodrgues@ucl.ac.uk). to uniformly sample and then report to the fusion center (FC). It is important to note that the actual physical environmental information is compressible not only in the time domain, due to the presence of intra-signal correlation, but also in the spatial domain, due to inter-signal correlation. However, it is in general difficult to conceive efficient compression schemes that account for both temporal and spatial correlation, duo to the distributed architecture of WSNs [4]. Various distributed coding techniques have been proposed in the literature [5]-[7], where SNs blindly compress their data with respect to others to reduce the communication cost in WSNs. However, the FC requires knowledge about the global correlation structure, or the capability to correctly track the correlation structure using some predictive model, to reconstruct the original data. This together with the fact that the signal correlation structure may change over time is difficult to be achieved in practice.

The conventional CS approach is based on the acquisition of a reduced number of samples via random projections in the time domain, and so no post compression process is required [8], [9]. There have been various generalizations of the conventional CS framework to scenarios where a group of signals exhibit both intra-signal and inter-signal correlation as in a WSN. For example, an extension of the conventional CS, named distributed CS [10], has been proposed to reconstruct a group of signals acquired by different SNs in a WSN, where these signals are assumed to satisfy some joint sparsity models. In [11], [12], the spatial signals obtained from different SNs are reconstructed using conventional CS with the assumption that the spatial sparsifying basis is known. This is difficult to be fulfilled when the global correlation structure varies in time. In [13]-[15], the authors propose to reconstruct a time sequence of signals one by one, using previous reconstruction results to enhance the current reconstruction. This method requires the encoder to have the ability to recover the first signal of the time sequence. In other words, one $\mathrm{SN}$ is required to sample at a higher rate than the other SNs, which leads to a shortened life time of that SN. In [16], the authors propose a data gathering method to reduce the communication cost of WSNs by using CS principles for inter-signal correlation. However, this method does not exploit the intra-signal correlation of each signal.

In this paper, we focus on a scenario where signals detected by a group of SNs have not only intra-signal correlation but also significant inter-signal correlation owing to some global factors and spatial correlations. Consequently, we propose a Fréchet mean based CS approach which together with CS 
principles leverages both intra-signal and inter-signal correlation to efficiently collect and/or reconstruct highly correlated signals in WSNs. In particular, the approach leads to a higher reconstruction quality for a certain target number of samples, or conversely, to a lower number of samples for a certain target reconstruction quality. Our contributions can be summarized as follows:

- We propose a method to estimate the sparse signal representation by calculating a Fréchet mean, or more importantly, a sparse Fréchet mean. This can be married to traditional sparse recovery algorithms to reduce the number of samples required for reconstruction. We also derive an upper bound of the sparse Fréchet mean estimation error to cast further insight onto the problem.

- We propose a penalized $\ell_{1}$ minimization algorithm where the sparse Fréchet mean is used appropriately to make $\ell_{1}$ minimization close to $\ell_{0}$ minimization, since fewer measurements are needed for exact reconstruction with $\ell_{0}$ minimization than with $\ell_{1}$ minimization [17].

- We propose a Fréchet mean enhanced greedy algorithm, called precognition matching pursuit (PMP), where the sparse Fréchet mean is used to estimate the support, i.e., the nonzero positions, of the sparse representation.

The advantage of our method is demonstrated by recovering data gathered by a WSN at the Intel Berkeley lab [18].

The following notation is used. Italic upper-case letters denote numbers, boldface upper-case letters denote matrices, boldface lower-case letters denote column vectors, and calligraphic upper-case letters denote support sets. The superscripts $(\cdot)^{T}$ and $(\cdot)^{\dagger}$ denote the transpose and pseudoinverse of a matrix, respectively. $\operatorname{rank}(\cdot)$ denotes the rank of a matrix. $x_{i}$ denotes the $i$ th element of $\mathbf{x} .\left\{\mathbf{X}_{k}\right\}$ and $\left\{\mathbf{x}_{k}\right\}$ $(k=1,2, \ldots, K)$ denote a group of matrices and vectors, respectively. $\overline{\mathcal{J}}$ denotes the complement of set $\mathcal{J}$. Now supp $\mathbf{x}$ denotes the set of nonzero elements of the vector $\mathbf{x}$ and $\operatorname{supp}(\mathbf{x}, S)$ denotes the set of the $S$ largest elements of the vector $\mathrm{x}$. By $\mathrm{x}_{\mathcal{J}}=M$, we mean that the elements of vector $\mathrm{x}$ whose indexes are in set $\mathcal{J}$ are set to be equal to $M$. $\|\mathbf{x}\|_{0}$ denotes the $\ell_{0}$ norm, i.e., the number of nonzero elements of the vector $\mathbf{x} .\|\mathbf{x}\|_{1}=\sum_{i}\left|x_{i}\right|$ denotes the $\ell_{1}$ norm of the vector $\mathbf{x} .\|\mathbf{x}\|_{2}=\sqrt{\sum_{i} x_{i}^{2}}$ denotes the $\ell_{2}$ norm of the vector $\mathbf{x}$.

\section{WSN SignAl ACQUisition BASED ON COMPRESSIVE SENSING}

\section{A. Conventional Compressive Sensing}

Consider a WSN scenario where a single $\mathrm{SN}$ acquires a certain signal to be sent to and reconstructed by a FC, based on conventional CS principles. In particular, the $\mathrm{SN}$ monitors a discrete signal $\mathbf{f} \in \mathbb{R}^{N}$ with a discrete representation $\mathbf{x} \in \mathbb{R}^{N}$ on some basis $\boldsymbol{\Psi} \in \mathbb{R}^{N \times N}$, so that $\mathbf{f}=\mathbf{\Psi} \mathbf{x}$. The signal is said to be sparse over the basis when $\|\mathbf{x}\|_{\ell_{0}} \leq S \ll N$.

The SN obtains a vector of measurements $\mathbf{y} \in \mathbb{R}^{M}(S<$ $M \ll N)$ via random projections, which typically involves a multiplication with a random sensing matrix $\Phi \in \mathbb{R}^{M \times N}$ as follows:

$$
\mathbf{y}=\boldsymbol{\Phi} \boldsymbol{\Psi}_{\mathbf{x}}+\mathbf{n}=\mathbf{A x}+\mathbf{n}
$$

where $\mathbf{n} \in \mathbb{R}^{M}$ denotes the measurement noise and $\mathbf{A}=$ $\boldsymbol{\Phi} \boldsymbol{\Psi} \in \mathbb{R}^{M \times N}$.

In general, it is not possible to recover the signal representation $\mathbf{x}$ from the signal measurements $\mathbf{y}$, because (1) represents an underdetermined system of linear equations. However, if $\mathbf{x}$ is known to be sparse, (1) is solvable under some conditions [8], [9]. The recovery procedure corresponds to the solution of the optimization problem given by:

$$
\min _{\hat{\mathbf{x}}}\|\hat{\mathbf{x}}\|_{0}, \quad \text { s.t. }\|\mathbf{A} \hat{\mathbf{x}}-\mathbf{y}\|_{2} \leq \epsilon,
$$

where $\epsilon>0$ is an estimate of the measurement noise level, or, in order to bypass the fact that such a problem is NP-hard, the optimization problem given by:

$$
\min _{\hat{\mathbf{x}}}\|\hat{\mathbf{x}}\|_{1}, \quad \text { s.t. } \quad\|\mathbf{A} \hat{\mathbf{x}}-\mathbf{y}\|_{2} \leq \epsilon .
$$

Note that the $\ell_{1}$ minimization problem in (3) represents a convex relaxation of the $\ell_{0}$ minimization problem in (2).

One of the widely used conditions that guarantees the successful reconstruction of $\mathbf{x}$ via solving (3) is the restricted isometry property (RIP).

Definition 1 (RIP): A matrix A of size $M \times N$ is said to satisfy the RIP of order $S$ with restricted isometry constant (RIC) $\delta_{S} \in(0,1)$ as the smallest number such that

$$
\left(1-\delta_{S}\right)\|\mathbf{x}\|_{2}^{2} \leq\|\mathbf{A} \mathbf{x}\|_{2}^{2} \leq\left(1+\delta_{S}\right)\|\mathbf{x}\|_{2}^{2}
$$

holds for all $\mathbf{x}$ with $\|\mathbf{x}\|_{0} \leq S$.

In the absence of measurement noise, it is established in [19] that any sparse signal with support size no larger than $S$ can be exactly recovered by solving (3) (with $\epsilon=0$ which implies $\mathbf{y}=\mathbf{A} \hat{\mathbf{x}})$ if the RIC satisfies $\delta_{2 S}<\sqrt{2}-1$. In the presence of measurement noise, it is also established in [19] that the reconstructed vector $\hat{\mathbf{x}}$ is a good approximation to the original signal representation $\mathbf{x}$, if $\mathbf{x}$ is sparse or nearly sparse.

Most of the reconstruction algorithms proposed in the literature can be classified into two categories: convex optimization algorithms and greedy algorithms. Generally speaking, convex optimization algorithms [20], [21] provide higher reconstruction performance in terms of number of measurements required than do greedy algorithms. However, greedy algorithms are also very attractive owing to their low computational complexity. In particular, some greedy algorithms such as Compressive Sampling Matching Pursuit (CoSaMP) [22] and Subspace Pursuit [23] deliver reconstruction performances which are very close to performances of convex optimization based approaches.

\section{B. Correlated Compressive Sensing (CCS)}

Consider now a WSN scenario where $K$ densely placed SNs acquire signals to be sent to and individually reconstructed by a FC (see Fig. 1). In particular, it is assumed that the signals sensed by these SNs exhibit both intra-sensor correlation and inter-sensor correlation. This correlated sensing model occurs for example in WSN applications that involve monitoring of various physical parameters, such as temperature, humidity, light intensity and air pressure. Global factors, e.g., the sun and prevailing winds, could result in the high correlation 


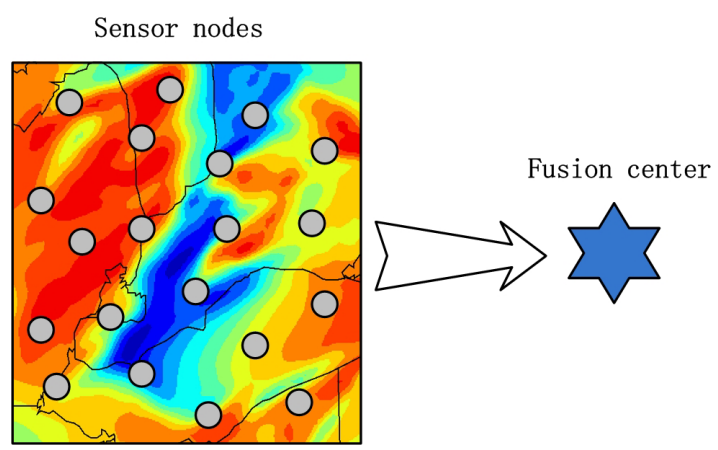

Fig. 1. A WSN with densely placed SNs. The adjacent SNs have highly correlated temperature values represented by colors.

between signals sensed by different SNs, that leads to physical parameters compressible in both the time and space domains.

The $k$ th $\mathrm{SN}$ monitors a discrete signal $\mathbf{f}_{k} \in \mathbb{R}^{N}$ with a discrete sparse representation $\mathbf{x}_{k} \in \mathbb{R}^{N}$ on some sparsifying basis $\boldsymbol{\Psi} \in \mathbb{R}^{N \times N}$ so that $\mathbf{f}_{k}=\mathbf{\Psi}_{k}$, where $\left\|\mathbf{x}_{k}\right\|_{0} \leq S$. The $k$ th SN also obtains a vector of measurement $\mathbf{y}_{k} \in \mathbb{R}^{M_{k}}(S<$ $\left.M_{k} \ll N\right)$ by calculating random projections with a randomly generated matrix $\boldsymbol{\Phi}_{k} \in \mathbb{R}^{M_{k} \times N}$ as follows:

$$
\mathbf{y}_{k}=\boldsymbol{\Phi}_{k} \boldsymbol{\Psi}_{\mathbf{x}_{k}}+\mathbf{n}_{k}=\mathbf{A}_{k} \mathbf{x}_{k}+\mathbf{n}_{k}
$$

where $\mathbf{n}_{k} \in \mathbb{R}_{k}^{M}$ denotes the measurement noise and $\mathbf{A}_{k}=$ $\boldsymbol{\Phi}_{k} \boldsymbol{\Psi} \in \mathbb{R}^{M_{k} \times N}$. The aim of correlated compressive sensing (CCS) is to leverage the intra-signal and inter-signal correlation of the signals in order to reduce the total number of SN samples (or random projections) to meet some target reconstruction performance.

\section{Prior Estimation of Correlated Sparse SignALS: A FrÉCHET MEAN APPROACH}

The key idea, in view of the fact that the physical signals sensed by different SNs exhibit high correlations that leads to similar sparse representations [10], is to infer a crude estimate of some sparse representation directly from the measurements, that can be leveraged to improve the signal reconstruction algorithms at the FC. In particular, we propose to exploit the Fréchet mean, or more importantly, the sparse Fréchet mean to estimate the sparse representation directly from the measurements prior to signal reconstruction.

\section{A. Fréchet Mean Estimation}

The Fréchet mean of $K$ sparse representations can be obtained from the measurements as follows:

$$
\tilde{\mathbf{x}}=\arg \min _{\tilde{\mathbf{x}}} \sum_{k=1}^{K} \lambda_{k} d^{2}\left(\mathbf{A}_{k} \tilde{\mathbf{x}}, \mathbf{y}_{k}\right),
$$

where $\lambda_{k}>0$ denotes the contribution weight of the $k$ th signal and $d\left(\mathbf{A}_{k} \tilde{\mathbf{x}}, \mathbf{y}_{k}\right)$ denotes the distance function between the vector $\mathbf{A}_{k} \tilde{\mathbf{x}}$ and $\mathbf{y}_{k}$.

The definition of the distance function should consider two aspects: i) it should be meaningful; and ii) it should lead to a simple solution of the optimization problem in (6). Therefore, we define the distance function as follows:

$$
d\left(\mathbf{A}_{k} \tilde{\mathbf{x}}, \mathbf{y}_{k}\right)=\left\|\mathbf{A}_{k} \tilde{\mathbf{x}}-\mathbf{y}_{k}\right\|_{2}^{2} .
$$

Consequently, the Fréchet mean can be obtained by solving the optimization problem:

$$
\min _{\tilde{\mathbf{x}}}\|\hat{\mathbf{A}} \tilde{\mathbf{x}}-\hat{\mathbf{y}}\|_{2}^{2},
$$

where the extended sensing matrix $\hat{\mathbf{A}} \in \mathbb{R}^{\left(\sum_{k=1}^{K} M_{k}\right) \times N}$ and the extended measurement vector $\hat{\mathbf{y}} \in \mathbb{R}^{\sum_{k=1}^{K} M_{k}}$ are given by $\hat{\mathbf{A}}=\left[\sqrt{\lambda_{1}} \mathbf{A}_{1}^{T}, \cdots, \sqrt{\lambda_{K}} \mathbf{A}_{K}^{T}\right]^{T}$ and $\hat{\mathbf{y}}=$ $\left[\sqrt{\lambda_{1}} \mathbf{y}_{1}^{T}, \cdots, \sqrt{\lambda_{K}} \mathbf{y}_{K}^{T}\right]^{T}$ respectively.

The Fréchet mean can be seen as a minimization of the sum of the squared Euclidean distances in low-dimensional spaces between the measurements and the common sparse representation. The weights $\lambda_{1}, \ldots, \lambda_{K}$ control the emphasis given to particular SN measurements. For example, if $\lambda_{1}=\ldots=\lambda_{K}$, equal emphasis is given to the measurement vectors in the calculation of the Fréchet mean. In contrast, if $\lambda_{k}>\lambda_{i}$ $(i \neq k)$, greater emphasis is given to the $k$ th measurement in the calculation of the Fréchet mean. This could lead to a better estimate of the $k$ th sparse representation.

The solution of the ordinary least squared optimization problem in (8) is given by:

$$
\tilde{\mathbf{x}}=\left(\hat{\mathbf{A}}^{H} \hat{\mathbf{A}}\right)^{-1} \hat{\mathbf{A}}^{H} \hat{\mathbf{y}},
$$

if $\operatorname{rank}(\hat{\mathbf{A}})=N$. This rank condition requires that $\sum_{k=1}^{K} M_{k} \geq N$ for randomly generated sensing matrices $\boldsymbol{\Phi}_{k}$ $(k=1, \ldots, K)$.

\section{B. Sparse Fréchet Mean Estimation}

It is more appropriate to consider a sparse version rather than the conventional version of the Fréchet mean as the estimate of the sparse signal representation in view of the fact that the signals are sparse. The sparse Fréchet mean $\tilde{\tilde{\mathbf{x}}}$ corresponds to the solution of the following optimization problem

$$
\min _{\tilde{\tilde{\mathbf{x}}}}\|\hat{\mathbf{A}} \tilde{\tilde{\mathbf{x}}}-\hat{\mathbf{y}}\|_{2}^{2}, \quad \text { s.t. } \quad\|\tilde{\tilde{\mathbf{x}}}\|_{0} \leq S .
$$

One common method to derive an approximate solution to the NP-hard problem in (10) involves the replacement of the $\ell_{0}$ norm by the $\ell_{1}$ norm. This gives rise to the optimization problem:

$$
\min _{\tilde{\tilde{\mathbf{x}}}}\|\hat{\mathbf{A}} \tilde{\tilde{\mathbf{x}}}-\hat{\mathbf{y}}\|_{2}^{2}, \quad \text { s.t. } \quad\|\tilde{\tilde{\mathbf{x}}}\|_{1} \leq \tilde{S},
$$

where $\tilde{S}>0$ represents the constraint on the $\ell_{1}$ norm of the Fréchet mean, which can be solved using the least-absolute shrinkage and selection operator (Lasso) [24] in polynomial time. A simpler method, which we propose to circumvent the high computational complexity associated with large dimensions, involves the determination of the sparse Fréchet mean $\tilde{\tilde{\mathbf{x}}}$ directly from the Fréchet mean $\tilde{\mathbf{x}}$, by forcing all but the $S$ 
largest components in absolute value of the Fréchet mean to be equal to zero, i.e.,

$$
\tilde{\tilde{x}}_{i}=\left\{\begin{array}{cl}
\tilde{x}_{i} & i \in \operatorname{supp}(\tilde{\mathbf{x}}, S) \\
0 & i \notin \operatorname{supp}(\tilde{\mathbf{x}}, S)
\end{array} .\right.
$$

(a)

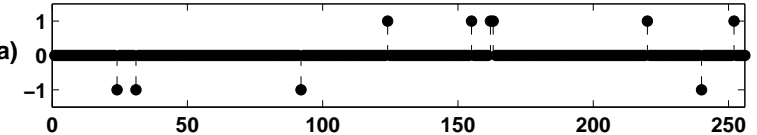

(b)

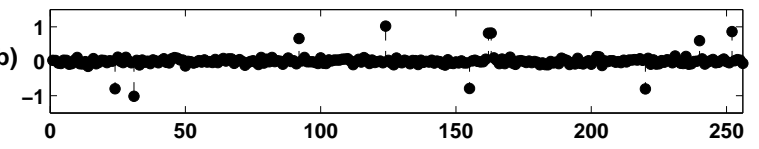

(c)

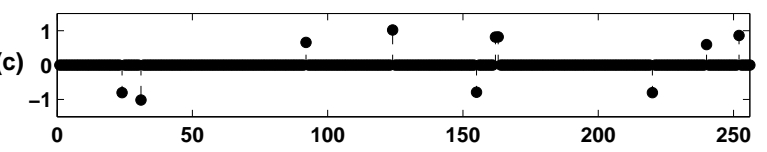

(d)

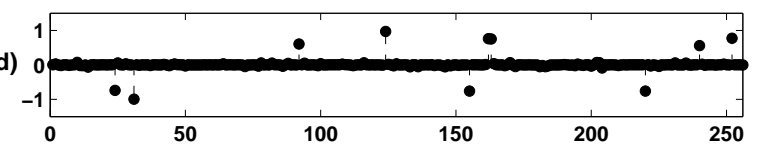

Fig. 2. Comparison of the sparse signal with the Fréchet mean. From top to bottom: (a) the sparse signal $\mathbf{x}$, (b) the Fréchet mean $\tilde{\mathbf{x}}$ in (8), (c) the sparse Fréchet mean $\tilde{\tilde{\mathbf{x}}}$ given by (12) and (d) the sparse Fréchet mean given by (11) The sparse representation $\mathbf{x}$ contains 10 nonzero components, all of which are \pm 1 spikes. The Fréchet mean is calculated from 11 sparse representations with $\lambda_{k}=1(k=1, \ldots, 11)$. All the representations are measured using i.i.d. random Gaussian matrices $\left(M_{1}=\ldots=M_{11}=100\right.$ and $\left.N=256\right)$.

Fig. 2 gives an example of the use of Fréchet mean and the sparse Fréchet mean. We generate $K=11$ correlated sparse representations $\mathbf{x}_{k}(k=1, \ldots K)$ with $N=256$ coefficients of which 246 are zero and 10 are \pm 1 random spikes as shown in the sub-figure (a) of Fig. 2. The inter-signal correlation in this experiment is modeled by generating 9 common spikes and 1 innovation spike. Random Gaussian matrices of size $100 \times 256$ are used as the equivalent sensing matrices $\mathbf{A}_{k}$ $(k=1, \ldots K)$. The Fréchet mean shown in the sub-figure (b) is calculated with the parameters $\lambda_{k}=1(k=1, \ldots K)$, which leads to a common Fréchet mean for all sparse representations. The sparse Fréchet means in the sub-figures (c) and (d) are calculated with the parameters $\lambda_{k}=1(k=1, \ldots K)$ by using (12) and (11) respectively. We note that the sparse Fréchet means are closer to the sparse representation of a randomly selected signal (out of the $K=11$ signals) than is the Fréchet mean, owing to to the fact that the sparsity constraint leads to the small estimation errors being equal to zeros. We also highlight that the sparse Fréchet mean only acts as a crude estimate of the sparse signal. Further refinements require the integration of the sparse Fréchet mean with sparse recovery algorithms (see Section IV).

\section{Estimation Error}

An important consideration relates to the squared error between the sparse Fréchet mean estimate of the sparse representation and the exact sparse representation. This is unveiled by the following Proposition.
Proposition 1: Consider the measurement model $\mathbf{y}_{k}=$ $\mathbf{A}_{k} \mathbf{x}_{k}+\mathbf{n}_{k}(k=1, \ldots, \mathrm{K})$, where the matrices $\mathbf{A}_{k}$ have RIC $\delta_{\mathrm{S}, k}$. Fix the integers $\mathrm{S}, \mathbf{S}_{d}$ and the positive numbers $\lambda_{k}(k=$ $1, \ldots, \mathrm{K})$. If $\left\|\mathbf{x}_{k}\right\|_{0} \leq \mathrm{S}(k=1, \ldots, \mathrm{K}),\left\|\mathbf{x}_{k_{1}}-\mathbf{x}_{k_{2}}\right\|_{0} \leq S_{d}{ }^{1}$ $\left(k_{1}, k_{2} \in\{1, \ldots, \mathrm{K}\}\right)$ and $\left\|\mathbf{n}_{k}\right\|_{2}^{2} \leq \epsilon^{2}(k=1, \ldots, \mathbf{K})$. Then the sparse Fréchet mean in (10) satisfies

$\left\|\tilde{\tilde{\mathbf{x}}}-\mathbf{x}_{k^{\prime}}\right\|_{2}^{2} \leq \sum_{k=1}^{K} \mathrm{C}_{1, k}\left\|\mathbf{x}_{k^{\prime}}-\mathbf{x}_{k}\right\|_{2}^{2}+\mathrm{C}_{2, k} \epsilon^{2}\left(k^{\prime}=1, \ldots, K\right)$,

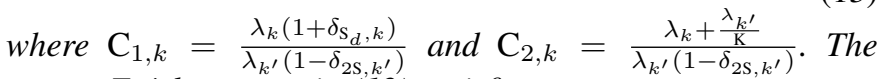
sparse Fréchet mean in (12) satisfies

$$
\begin{array}{r}
\left\|\tilde{\tilde{\mathbf{x}}}-\mathbf{x}_{k^{\prime}}\right\|_{2}^{2} \leq \sum_{k=1}^{K} \mathrm{C}_{1, k}\left\|\mathbf{x}_{k^{\prime}}-\mathbf{x}_{k}\right\|_{2}^{2}+\mathrm{C}_{2, k} \epsilon^{2}+\mathrm{C}_{3, k} \tilde{\epsilon}^{2} \\
\left(k^{\prime}=1, \ldots, K\right),
\end{array}
$$
where $\mathrm{C}_{3, k}=\frac{\lambda_{k}}{\lambda_{k^{\prime}}\left(1-\delta_{2 \mathrm{~s}, k^{\prime}}\right)}$ and $\tilde{\epsilon}^{2} \geq\left\|\mathbf{A}_{k}(\tilde{\tilde{\mathbf{x}}}-\tilde{\mathbf{x}})\right\|_{2}^{2}$
$(k=1, \ldots, \mathrm{K})$.

Proof: We first consider the sparse Fréchet mean in (10). Since $\tilde{\tilde{\mathbf{x}}}$ solves the optimization problem in (10), where the sparsity level of $\tilde{\tilde{\mathbf{x}}}$ is equal to the sparsity level of $\mathbf{x}_{k}(k=$ $1, \ldots, K)$, then we have

$$
\sum_{k=1}^{K} \lambda_{k}\left\|\mathbf{A}_{k} \tilde{\tilde{\mathbf{x}}}-\mathbf{y}_{k}\right\|_{2}^{2} \leq \sum_{k=1}^{K} \lambda_{k}\left\|\mathbf{A}_{k} \mathbf{x}_{k^{\prime}}-\mathbf{y}_{k}\right\|_{2}^{2}
$$

for any $k^{\prime}$. Therefore, according to the RIP in (4), the triangle inequality, the inequality in (15) and the assumptions, we can derive through the following chain of inequalities that

$$
\begin{aligned}
& \left\|\tilde{\tilde{\mathbf{x}}}-\mathbf{x}_{k^{\prime}}\right\|_{2}^{2} \leq \frac{1}{1-\delta_{2 S, k^{\prime}}}\left\|\mathbf{A}_{k^{\prime}}\left(\tilde{\tilde{\mathbf{x}}}-\mathbf{x}_{k^{\prime}}\right)\right\|_{2}^{2} \\
& \leq \frac{1}{1-\delta_{2 S, k^{\prime}}}\left\|\mathbf{A}_{k^{\prime}} \tilde{\tilde{\mathbf{x}}}-\mathbf{y}_{k^{\prime}}\right\|_{2}^{2}+\frac{\left\|\mathbf{n}_{k^{\prime}}\right\|_{2}^{2}}{1-\delta_{2 S, k^{\prime}}} \\
& \leq \frac{1}{1-\delta_{2 S, k^{\prime}}} \sum_{k=1}^{K} \frac{\lambda_{k}}{\lambda_{k^{\prime}}}\left\|\mathbf{A}_{k} \tilde{\tilde{\mathbf{x}}}-\mathbf{y}_{k}\right\|_{2}^{2}+\frac{\epsilon^{2}}{1-\delta_{2 S, k^{\prime}}} \\
& \leq \frac{1}{1-\delta_{2 S, k^{\prime}}} \sum_{k=1}^{K} \frac{\lambda_{k}}{\lambda_{k^{\prime}}}\left\|\mathbf{A}_{k} \mathbf{x}_{k^{\prime}}-\mathbf{y}_{k}\right\|_{2}^{2}+\frac{\epsilon^{2}}{1-\delta_{2 S, k^{\prime}}} \\
& \leq \frac{1}{1-\delta_{2 S, k^{\prime}}} \sum_{k=1}^{K} \frac{\lambda_{k}}{\lambda_{k^{\prime}}}\left(\left\|\mathbf{A}_{k}\left(\mathbf{x}_{k^{\prime}}-\mathbf{x}_{k}\right)\right\|_{2}^{2}+\left\|\mathbf{n}_{k}\right\|_{2}^{2}\right)+\frac{\epsilon^{2}}{1-\delta_{2 S, k^{\prime}}} \\
& \leq \sum_{k=1}^{K} \frac{\lambda_{k}\left(1+\delta_{S_{d}, k}\right)}{\lambda_{k^{\prime}}\left(1-\delta_{2 S, k^{\prime}}\right)}\left\|\mathbf{x}_{k^{\prime}}-\mathbf{x}_{k}\right\|_{2}^{2}+\frac{\epsilon^{2}}{1-\delta_{2 S, k^{\prime}}}\left(1+\sum_{k=1}^{K} \frac{\lambda_{k}}{\lambda_{k^{\prime}}}\right) .
\end{aligned}
$$

We now consider the the sparse Fréchet mean $\tilde{\tilde{\mathbf{x}}}$ in (12). Since $\tilde{\mathbf{x}}$ solves the optimization problem in (8) then

$$
\sum_{k=1}^{K} \lambda_{k}\left\|\mathbf{A}_{k} \tilde{\mathbf{x}}-\mathbf{y}_{k}\right\|_{2}^{2} \leq \sum_{k=1}^{K} \lambda_{k}\left\|\mathbf{A}_{k} \mathbf{x}_{k^{\prime}}-\mathbf{y}_{k}\right\|_{2}^{2}
$$

${ }^{1}$ This assumption arises from the fact that these sparse signals have considerable inter-signal correlations. 
for any $k^{\prime}$. Therefore, by following the previous procedure, we can also derive through the following sequence of inequalities that

$$
\begin{aligned}
& \left\|\tilde{\tilde{\mathbf{x}}}-\mathbf{x}_{k^{\prime}}\right\|_{2}^{2} \leq \frac{1}{1-\delta_{2 S, k^{\prime}}}\left\|\mathbf{A}_{k^{\prime}}\left(\tilde{\tilde{\mathbf{x}}}-\mathbf{x}_{k^{\prime}}\right)\right\|_{2}^{2} \\
& \leq \frac{1}{1-\delta_{2 S, k^{\prime}}}\left\|\mathbf{A}_{k^{\prime}} \tilde{\tilde{\mathbf{x}}}-\mathbf{y}_{k^{\prime}}\right\|_{2}^{2}+\frac{\left\|\mathbf{n}_{k^{\prime}}\right\|_{2}^{2}}{1-\delta_{2 S, k^{\prime}}} \\
& \leq \frac{1}{1-\delta_{2 S, k^{\prime}}}\left(\left\|\mathbf{A}_{k^{\prime}} \tilde{\mathbf{x}}-\mathbf{y}_{k^{\prime}}\right\|_{2}^{2}+\left\|\mathbf{A}_{k^{\prime}}(\tilde{\tilde{\mathbf{x}}}-\tilde{\mathbf{x}})\right\|_{2}^{2}\right)+\frac{\epsilon^{2}}{1-\delta_{2 S, k^{\prime}}} \\
& \leq \frac{1}{1-\delta_{2 S, k^{\prime}}} \sum_{k=1}^{K} \frac{\lambda_{k}}{\lambda_{k^{\prime}}}\left(\left\|\mathbf{A}_{k} \tilde{\mathbf{x}}-\mathbf{y}_{k}\right\|_{2}^{2}+\left\|\mathbf{A}_{k}(\tilde{\tilde{\mathbf{x}}}-\tilde{\mathbf{x}})\right\|_{2}^{2}\right) \\
& +\frac{\epsilon^{2}}{1-\delta_{2 S, k^{\prime}}} \\
& \leq \frac{1}{1-\delta_{2 S, k^{\prime}}} \sum_{k=1}^{K} \frac{\lambda_{k}}{\lambda_{k^{\prime}}}\left(\left\|\mathbf{A}_{k} \mathbf{x}_{k^{\prime}}-\mathbf{y}_{k}\right\|_{2}^{2}+\left\|\mathbf{A}_{k}(\tilde{\tilde{\mathbf{x}}}-\tilde{\mathbf{x}})\right\|_{2}^{2}\right) \\
& +\frac{\epsilon^{2}}{1-\delta_{2 S, k^{\prime}}} \\
& \leq \frac{1}{1-\delta_{2 S, k^{\prime}}} \sum_{k=1}^{K} \frac{\lambda_{k}}{\lambda_{k^{\prime}}}\left(\left\|\mathbf{A}_{k} \mathbf{x}_{k^{\prime}}-\mathbf{x}_{k}\right\|_{2}^{2}+\left\|\mathbf{n}_{k}\right\|_{2}^{2}\right. \\
& \left.+\left\|\mathbf{A}_{k}(\tilde{\tilde{\mathbf{x}}}-\tilde{\mathbf{x}})\right\|_{2}^{2}\right)+\frac{\epsilon^{2}}{1-\delta_{2 S, k^{\prime}}} \\
& \leq \sum_{k=1}^{K} \frac{\lambda_{k}\left(1+\delta_{S_{d}, k}\right)}{\lambda_{k^{\prime}}\left(1-\delta_{2 S, k^{\prime}}\right)}\left\|\mathbf{x}_{k^{\prime}}-\mathbf{x}_{k}\right\|_{2}^{2}+\frac{\epsilon^{2}}{1-\delta_{2 S, k^{\prime}}}\left(1+\sum_{k=1}^{K} \frac{\lambda_{k}}{\lambda_{k^{\prime}}}\right) \\
& +\frac{1}{1-\delta_{2 S, k^{\prime}}} \sum_{k=1}^{K} \frac{\lambda_{k}}{\lambda_{k^{\prime}}}\left\|\mathbf{A}_{k}(\tilde{\tilde{\mathbf{x}}}-\tilde{\mathbf{x}})\right\|_{2}^{2} .
\end{aligned}
$$

Proposition 1 gives the upper bound of the squared pairwise Euclidean distance between the sparse Fréchet mean of the sparse representations and a target sparse representation. The bound (13) is sharp in the noiseless case $\epsilon=0$ and all signals considered are the same, i.e. $\mathbf{x}_{k}=\mathbf{x}_{k^{\prime}}\left(\forall k, k^{\prime}\right)$. In this case, the sparse Fréchet mean is equal to the target signal. If the signals are slightly different, then the squared estimation error bound (13) is equal to a sum of weighed distances between the target signal and other signals plus a noise related term. Note that the additional term, i.e., $C_{3, k} \tilde{\epsilon}^{2}$, in the bound (14) reveals the estimation error penalty due to the calculation of the sparse Fréchet mean via (12).

\section{Correlated Sparse Signal Reconstruction ALGORITHMS}

We now leverage the availability of the Fréchet mean to propose an enhanced convex algorithm, i.e., the penalized $\ell_{1}$ minimization, and an enhanced greedy algorithm, i.e., the PMP, to reconstruct a group of $K$ correlated sparse signals. These signals are sensed in the CS manner by the SNs and reconstructed one by one at the FC based on the signal measurements $\mathbf{y}_{k}(k=1, \ldots, K)$, and the signal sparse Fréchet mean $\tilde{\tilde{\mathbf{x}}}_{k}(k=1, \ldots, K)$. Note that it is possible to use a specialized Fréchet mean, where $\lambda_{k^{\prime}}>\lambda_{k}\left(\forall k, k^{\prime}\right)$ to reconstruct the $k^{\prime}$ th signal. This requires the calculation of a Fréchet mean for each signal, but also leads to a lower estimation error bound in view of Proposition 1. Alternatively, it is possible to use a single common Fréchet mean, where $\lambda_{1}=\ldots=\lambda_{K}$, to reconstruct each signal. This only requires the calculation of a single Fréchet mean, but also lead to a higher estimation error bound.

\section{A. Penalized $\ell_{1}$ Minimization Algorithm}

The penalized $\ell_{1}$ minimization algorithm uses the sparse Fréchet mean $\tilde{\tilde{\mathbf{x}}}_{k}$ of the sparse representation $\mathbf{x}_{k}$ as a penalty vector in the $\ell_{1}$ minimization algorithm. The reconstruction corresponds to the solution of the optimization problem:

$$
\min _{\hat{\mathbf{x}}_{k}}\left\|\mathbf{W}_{k} \hat{\mathbf{x}}_{k}\right\|_{1} \quad \text { s.t. }\left\|\mathbf{A}_{k} \hat{\mathbf{x}}_{k}-\mathbf{y}_{k}\right\|_{2} \leq \epsilon,
$$

where $\mathbf{W}_{k}=\operatorname{diag}\left(\frac{1}{\left|\tilde{\tilde{x}}_{k, i}\right|+\beta}\right) \in \mathbb{R}^{N \times N}$ is a diagonal matrix which contains a set of penalties. The parameter $\beta>0$ is a very small positive number to ensure that $\left|\tilde{\tilde{x}}_{k, i}\right|+\beta \neq 0$.

The motivation of the approach relates to the fact that the $\ell_{1}$-norm $\left\|\mathbf{W}_{k} \hat{\mathbf{x}}_{k}\right\|_{1}$ in (19) is a better approximation to the $\ell_{0}$ norm than the $\ell_{1}$-norm $\left\|\hat{\mathbf{x}}_{k}\right\|_{1}$ in (3) [17] because $\left\|\mathbf{W}_{k} \hat{\mathbf{x}}_{k}\right\|_{1} \approx$ $\left\|\hat{\mathbf{x}}_{k}\right\|_{0}$ for a good prior estimate $\tilde{\tilde{\mathbf{x}}}_{k}$. Consequently, one expects the algorithm in (19) to outperform the algorithm in (3). The pseudo-code for penalized $\ell_{1}$ minimization for a sequence of signals is presented in Algorithm 1. The value of $\beta$ should be small enough to lead to $\frac{\hat{x}_{k, i}}{\left|\tilde{\tilde{x}}_{k, i}\right|+\beta} \approx 1$ for a good prior estimate $\tilde{\tilde{\mathbf{x}}}_{k}$. This can be fulfilled for example by letting $\beta=$ $\frac{1}{100} \min _{i}\left\{\tilde{\tilde{x}}_{k, i} \mid \tilde{\tilde{x}}_{k, i} \neq 0\right\}$.

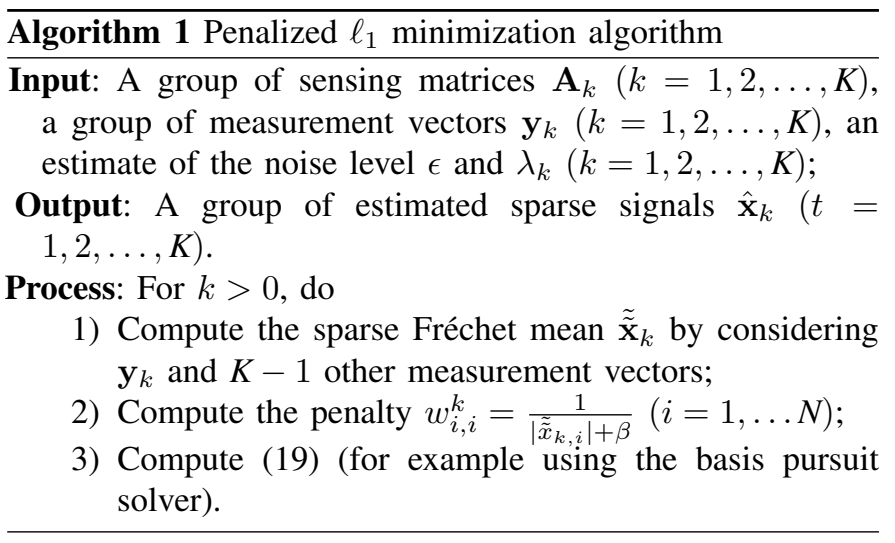

\section{B. Precognition Matching Pursuit (PMP)}

The greedy algorithm, namely PMP, aims to achieve fast reconstruction and a reduced requirement on the number of measurements compared to traditional matching pursuit (MP) and its variations [23], [25], [26]. In particular, the PMP algorithm uses the support of the sparse Fréchet mean as a prior estimate of the sparse representation support. We denote the support of the sparse Fréchet mean for the $k$ th signal by $\tilde{\mathcal{J}}_{k}$.

The key idea of the PMP is to find a support $\mathcal{J}_{k}$ that is closest to the actual support of the $k$ th signal $\mathbf{x}_{k}$. Then, 
the reconstructed signal $\hat{\mathbf{x}}_{k}$ is calculated by solving the least square optimization problem:

$$
\min _{\hat{\mathbf{x}}_{k}}\left\|\mathbf{A}_{k, \mathcal{J}_{k}} \hat{\mathbf{x}}_{k, \mathcal{J}_{k}}-\mathbf{y}_{k}\right\|_{2}^{2},
$$

which leads to $\hat{\mathbf{x}}_{k}=\mathbf{A}_{k, \mathcal{J}_{k}}^{\dagger} \mathbf{y}_{k}$. The PMP algorithm involves: an initialization phase, where a residual signal $\mathbf{r}$ and the estimated signal $\hat{\mathbf{x}}_{k}$ are initialized, and an iterative phase, where the support of the actual signal is iteratively updated. In particular, the iterative phase includes the steps:

1) Estimation of the support of the residual signal and of the estimated signal from the previous iteration (see $2 \mathrm{a}$ and $2 b$ in algorithm 2);

2) update of the signal support (see $2 \mathrm{c}$ in algorithm 2);

3) update of the residual signal and the estimated signal (see $2 \mathrm{~d}$ and $2 \mathrm{e}$ in algorithm 2).

The estimated signal support $\mathcal{J}_{k}$ in each iteration is composed of the sparse Fréchet mean support $\tilde{\mathcal{J}}_{k}$, the $L=$ $2 S-\left\|\tilde{\mathcal{J}}_{k}\right\|_{0}$ largest values of a residual vector returned in the previous iteration, and the estimated signal support returned in the previous iteration. The iterations stop when the result satisfies some halting condition. For example, one can either set a threshold for the the residual's Frobenius norm or let the algorithm stop when the improvement of the residual is smaller than a threshold based on empirical knowledge. One can observe that the PMP is similar to the CoSaMP [22] except for the additional sparse Fréchet mean support in each iteration. This key innovation enables the PMP to return a better reconstructed signal. The pseudo-code of the PMP for a sequence of signals is described in Algorithm 2.

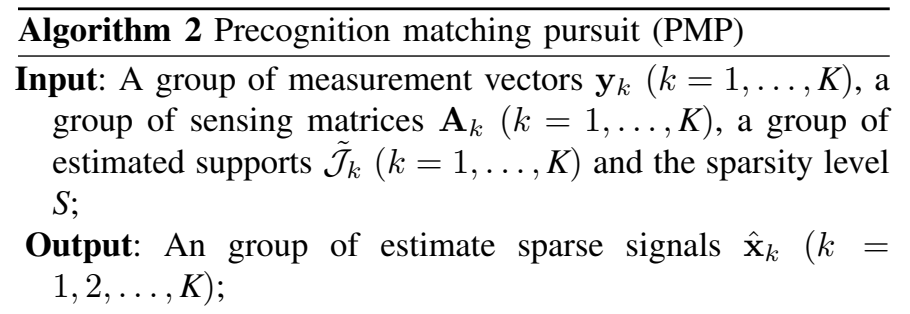

Process: For $k>0$, do

1) Initialization: Let $\hat{\mathbf{x}}_{k}=0$ and $\mathbf{r}=\mathbf{y}_{k}$;

2) Repeat:
a) $\mathbf{u}=\mathbf{A}_{k}^{T} \mathbf{r}, L=2 S-\left\|\tilde{\mathcal{J}}_{k}\right\|_{0}$;
b) $\mathcal{T}=\operatorname{suppu}_{L}, \mathcal{O}=\operatorname{supp} \hat{\mathbf{x}}_{k}$;
c) $\mathcal{J}_{k}=\mathcal{T} \cup \tilde{\mathcal{J}}_{k} \cup \mathcal{O}$;
d) $\mathbf{v}=\mathbf{A}_{k, \mathcal{J}_{k}}^{\dagger} \mathbf{y}_{k}, \mathcal{Q}=\operatorname{supp}_{S}$
e) $\hat{\mathbf{x}}_{k, \mathcal{Q}}=\mathbf{v}_{\mathcal{Q}}, \hat{\mathbf{x}}_{k, \overline{\mathcal{Q}}}=0, \mathbf{r}=\mathbf{y}_{k}-\mathbf{A}_{k, \mathcal{J}_{k}} \hat{\mathbf{x}}_{k}$;
f) if halting condition true then quit the iteration; end if.

We note that the PMP requires the knowledge of the sparsity level $S$, which is also required by many other MP algorithms, such as orthogonal matching pursuit (OMP), regularized orthogonal matching pursuit (ROMP) and CoSaMP. For example, one can use some empirical knowledge about the sparsity level $S$, or else one can run the PMP algorithm with several different sparsity levels to choose the signal with minimal squared error $\left\|\mathbf{A}_{k} \hat{\mathbf{x}}_{k}-\mathbf{y}_{k}\right\|_{2}^{2}$ as the final solution.

\section{EXPERIMENTAL RESUlts}

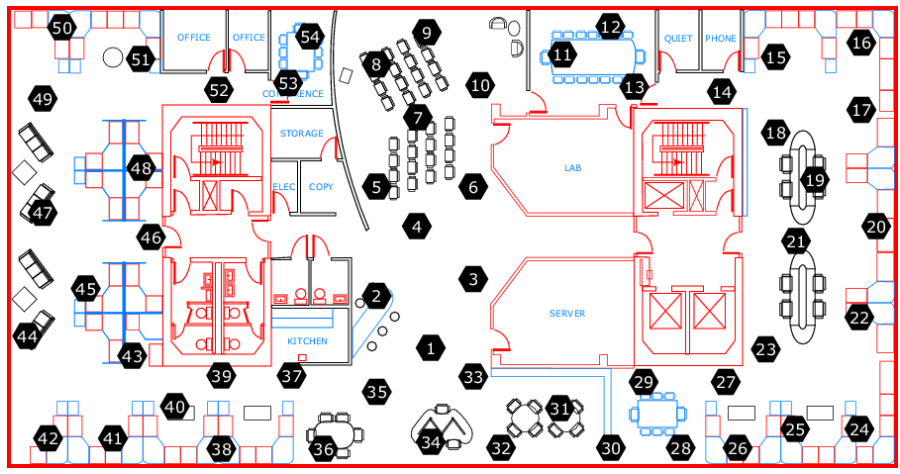

Fig. 3. Layout of the WSN of the Intel Berkeley Research lab.

We now apply the proposed method to reconstruct the signals gathered by the WSN located in the Intel Berkeley Research lab [18]. In this WSN, 54 Mica2Dot SNs with weather boards were deployed in one floor of the lab building as shown in Fig. 3. Each SN had measured the environmental humidity, temperature and light level for more than one month.

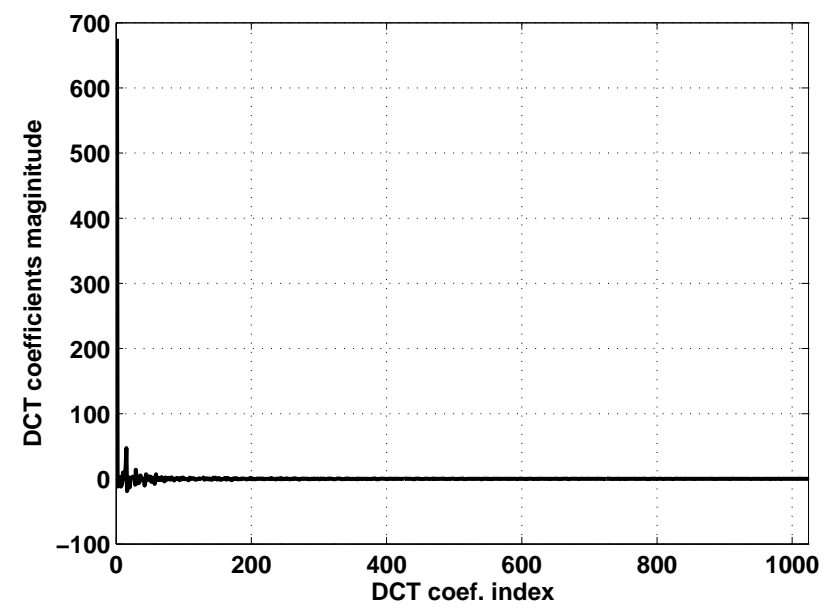

Fig. 4. DCT coefficients of a typical temperature signal detected by the WSN.

Note that the signals monitored by the WSN might be compressible rather than exactly sparse in some sparsifying basis, and all the measurements are disturbed by the noise. In the following evaluation of our method, we use the discrete cosine transform (DCT) as the sparsifying domain. When we investigated the data collected by these SNs, we noticed that some of the SNs data experienced a high level of loss. Thus, we only used the contiguous data that was available from $K=19$ of the SNs in our experiment. In Fig. 4, we show that the DCT coefficients of the temperature signal sensed by the $1 \mathrm{st} \mathrm{SN}$, are very sparse. All the other signals also have similarly sparse DCT vectors. We also note that the environmental temperature signals in the detected area have high spatial correlations as shown in Fig. 5. Instead of uniform sampling, each SN independently and randomly collects a small portion of the original samples and transmits them to 


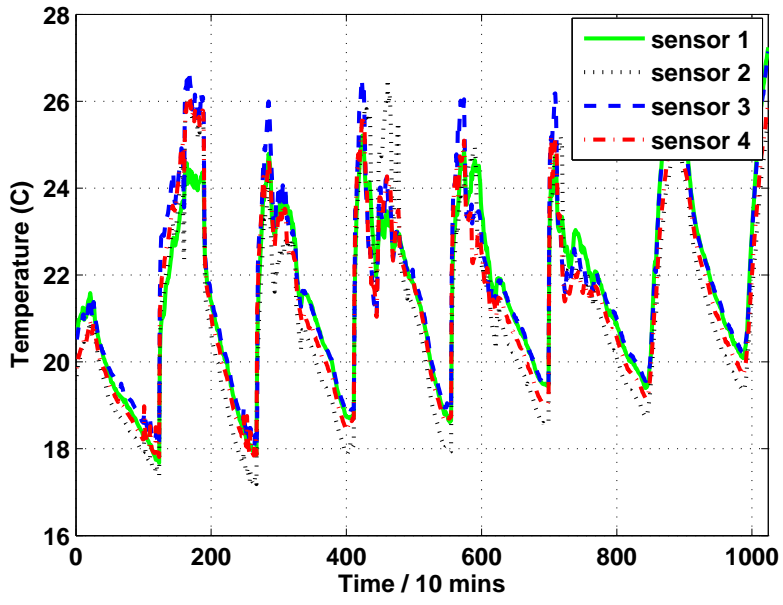

Fig. 5. Environmental temperature signals detected by the WSN.

the FC. Therefore, the sensing matrices $\mathbf{A}_{k}$ become random partial DCT matrices.
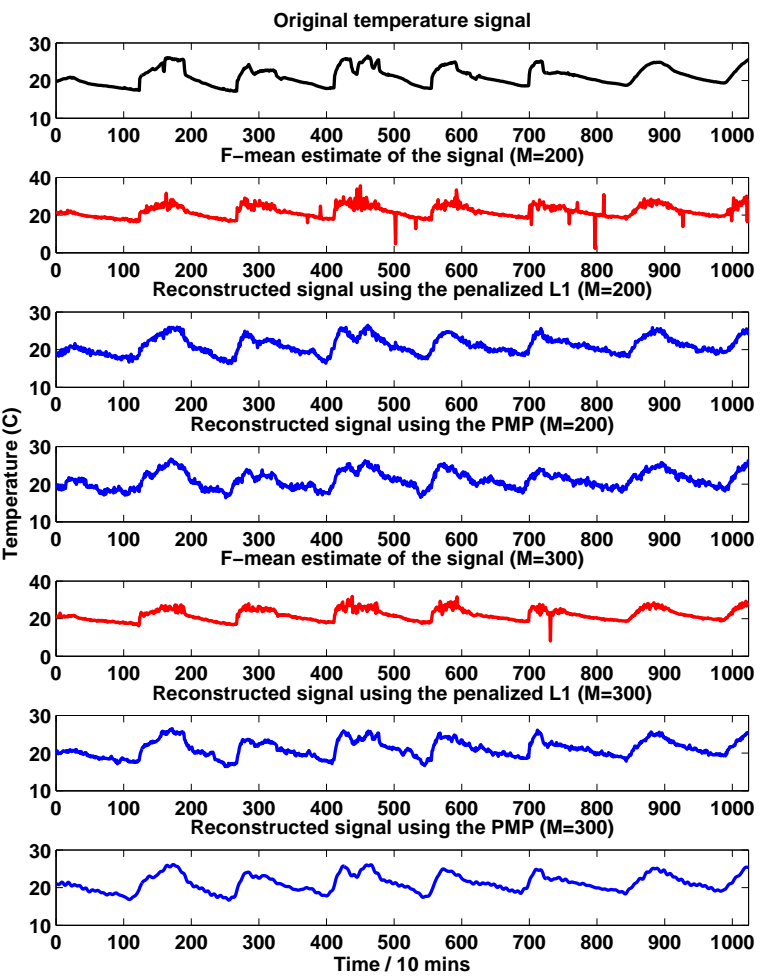

Fig. 6. Environmental temperature signal detected by the 5th $\mathrm{SN}$ and reconstruction results.

All the signals we employ in the following study have a length of $N=1024$. We let the sparsity level be $S=\frac{N}{20}$ in our simulation $^{3}$. We calculate the Fréchet mean from the randomly sampled signal of different SNs with $\lambda_{1}=\ldots=\lambda_{K}=1$ for

${ }^{3}$ In practice, the value of sparsity level is often based on empirical knowledge. all the signals, and then derive the common sparse Fréchet mean $\tilde{\tilde{\mathbf{x}}}_{k}$ by using (8) and (12). The sparse Fréchet mean is used as an input to the penalized $\ell_{1}$ minimization, where we let $\beta=\frac{1}{100} \min _{i}\left\{\tilde{\tilde{x}}_{k, i} \mid \tilde{\tilde{x}}_{k, i} \neq 0\right\}$, and the PMP is used to independently reconstruct each signal. In Fig. 6, we demonstrate the effectiveness of our method by comparing the original temperature signal of the 5th SN with the reconstructed signal. All the other temperature signals show a similarly close match between the reconstructed and the original signals.
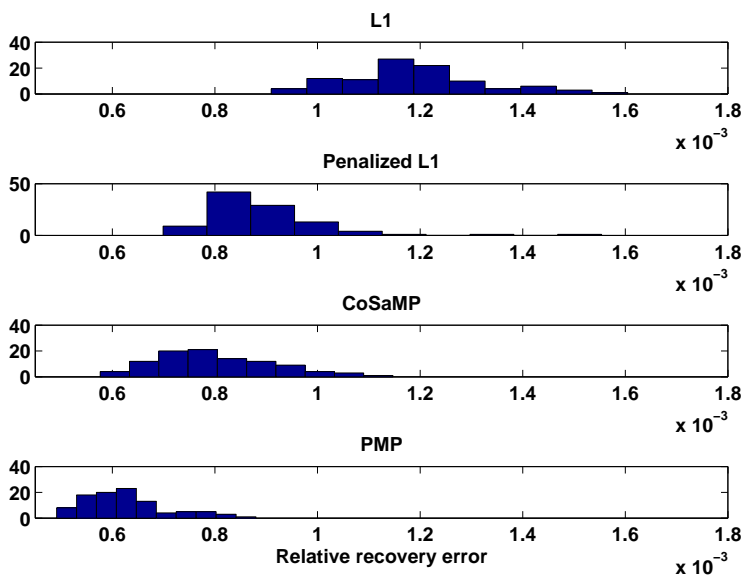

Fig. 7. Histogram of the relative recovery error of the reconstructed temperature signal of the 5th $\mathrm{SN}$ with different reconstruction algorithms $(M=300$ and $N=1024)$.

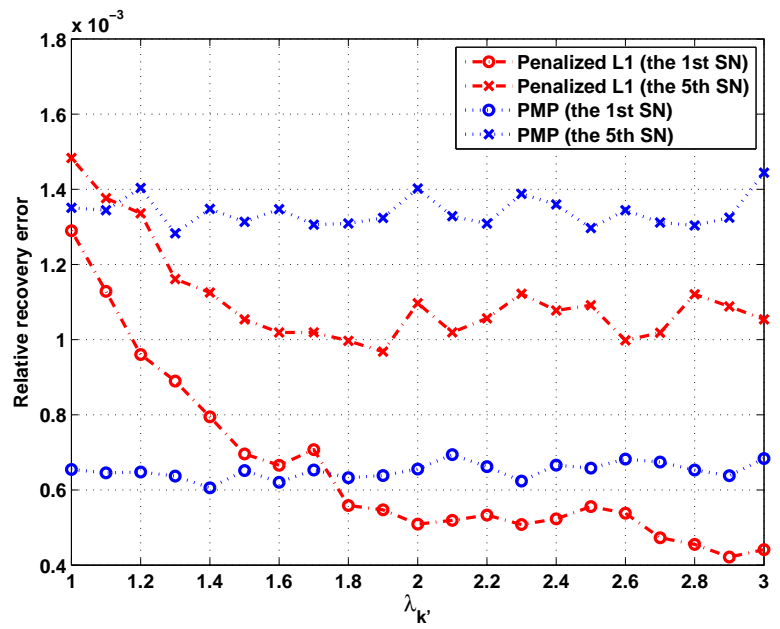

Fig. 8. Environmental temperature signal reconstruction performance of using different choices of $\lambda_{k^{\prime}}(M=200$ and $N=1024)$.

The sparse Fréchet mean estimate of the signal is also plotted to highlight that the result of the reconstruction algorithms exhibit further refinement. Now, we define the relative recovery error for a single $\mathrm{SN}$ to be equal to $\frac{\left\|\hat{\mathbf{f}}_{k}-\mathbf{f}_{k}\right\|_{2}^{2}}{\left\|\mathbf{f}_{k}\right\|_{2}^{2}}$, where $\mathbf{f}_{k}$ and $\hat{\mathbf{f}}_{k}$ are the original signal and the reconstructed signal of the $k$ th SN respectively. In Fig. 7 we illustrate as an example the distribution of the relative recovery error for the 5th SN. We let $\lambda_{k}=1(k=1, \ldots, K)$ in the calculation of the sparse 
TABLE I

THE SUCCESSFUl RECONSTRUCTION PROBABILITIES OF THE 1ST SN AND THE 5TH SN WITH DIFFERENT ALGORITHMS $(N=1024)$.

\begin{tabular}{cccccc}
\hline \hline & & The 1st SN & The 1st SN & The 5th SN & The 5th SN \\
& & & Th \\
& & $87.3 \%$ & $M=250$ & $M=300$ & $M=250$ \\
\hline L1 & & $15.1 \%$ & $5.5 \%$ & $0.0 \%$ \\
Penalized L1 & $\lambda_{k^{\prime}}=1$ & $94.6 \%$ & $57.4 \%$ & $86.0 \%$ & $16.5 \%$ \\
Penalized L1 & $\lambda_{k^{\prime}}=1.5$ & $98.8 \%$ & $95.8 \%$ & $95.7 \%$ & $82.0 \%$ \\
CoSaMP & & $99.56 \%$ & $96.38 \%$ & $92.44 \%$ & $33.26 \%$ \\
PMP & $\lambda_{k^{\prime}}=1$ & $99.95 \%$ & $99.82 \%$ & $99.37 \%$ & $87.33 \%$ \\
PMP & $\lambda_{k^{\prime}}=1.5$ & $99.96 \%$ & $99.85 \%$ & $99.38 \%$ & $87.52 \%$ \\
\hline
\end{tabular}

Fréchet mean. It is clear that the performance of the proposed two algorithms, i.e., the Penalized $\ell_{1}$ minimization and the PMP, surpass the performance of the two conventional algorithms, i.e., the $\ell_{1}$ minimization and the CoSaMP, respectively. In Fig. 8, we also show the reconstruction performance of temperature signals of the 1 st $\mathrm{SN}$ and the 5 th $\mathrm{SN}$ with different choices of $\left\{\lambda_{k}\right\}$ in the sparse Fréchet mean calculation, and the results are averaged over 100 trials. In particular, we let all $\lambda_{k}=1(k=1, \ldots, K)$ except for the value $\lambda_{k^{\prime}}$ corresponding to the target $k^{\prime}$ th SN. Note that the reconstruction performance of the penalized $\ell_{1}$ minimization algorithm can be improved by using $\lambda_{k^{\prime}}$ greater than 1 . However, the best choices of $\lambda_{k^{\prime}}$ value for the 1st SN and the 5th SN are different. Although one cannot find a unique value of $\lambda_{k^{\prime}}$ which is best for all SNs, it worth choosing a value of $\lambda_{k^{\prime}}$ slightly greater than 1 in order to obtain some performance gain. On the other hand, we note that the reconstruction performance of the PMP appears not to be significantly affected by different choices of $\lambda_{k^{\prime}}$. By defining a successful reconstruction as one where the relative recovery error is less than $10^{-3}$, we investigate the probability of successful reconstruction of a single SN with different algorithms. We let all $\lambda_{k}=1$ $(k=1, \ldots, K)$ except for the value $\lambda_{k^{\prime}}$ corresponding to the target $k^{\prime}$ th SN in the calculation of the sparse Fréchet mean. The reconstruction probability results are shown in Table I. Note that the reconstruction probabilities can be improved by using the Fréchet mean enhanced algorithms, i.e., the penalized $\ell_{1}$ minimization algorithm and the PMP algorithm.

We define the relative recovery error for multiple SNs in a WSN to be $\frac{\sum_{k=1}^{K}\left\|\hat{\mathbf{f}}_{k}-\mathbf{f}_{k}\right\|_{2}^{2}}{\sum_{k=1}^{K}\left\|\boldsymbol{f}_{k}\right\|_{2}^{2}}$. In Fig. 9, we show the superiority of the Fréchet mean enhanced algorithms, i.e., the penalized $\ell_{1}$ minimization algorithm and the PMP, in comparison with the $\ell_{1}$ minimization algorithm and the CoSaMP algorithm which is one of the most efficient greedy algorithms. In particular, we consider the use of a specialized sparse Fréchet mean, where $\lambda_{k^{\prime}}=1.5$ for the target signal and $\lambda_{k}=1$ for the other signals, as well as a common sparse Fréchet mean where $\lambda_{1}=\ldots=\lambda_{K}=1$. The results are averaged over 100 trials. We use the CVX code (http://www.stanford.edu/ boyd/cvx/) to solve the penalized $\ell_{1}$ minimization problem. The penalized $\ell_{1}$ minimization algorithm takes several minutes to reconstruct the group of signals while greedy algorithms just requires a few seconds using the same PC, which makes greedy algorithms promising in terms of computational complexity. We also observe that the performance of algorithms that employ the specialized sparse Fréchet mean is also better than that of algorithms that use the common sparse Fréchet mean.

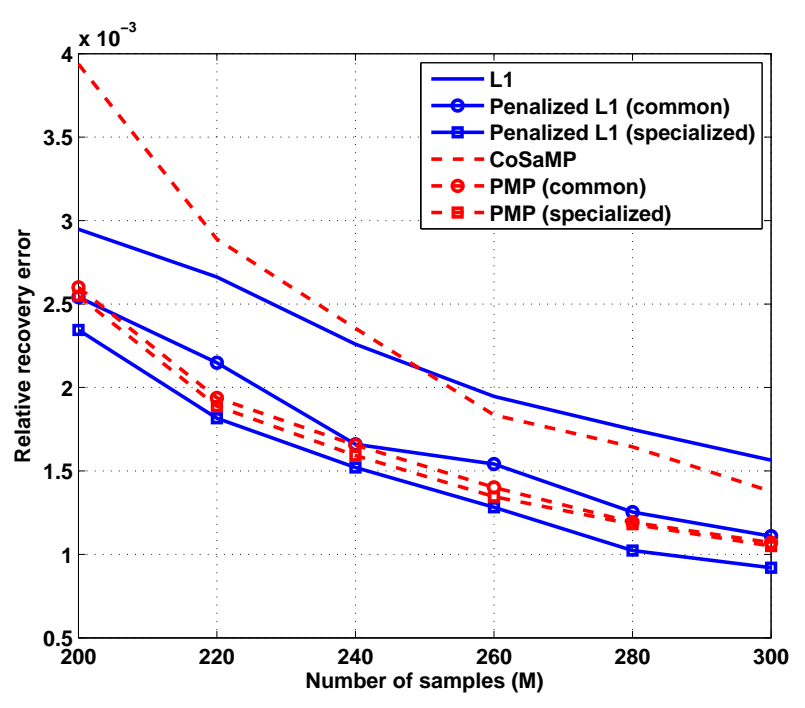

Fig. 9. Performance of three different reconstruction algorithms for temperature signals $(N=1024)$ detected by the WSN.

\section{CONCLUSIONS}

In this paper, we proposed a Fréchet mean enhanced CS approach to efficiently monitor the environmental physical parameters by WSNs. The proposed approach leads to a considerable reduction in the number of samples required by the conventional CS framework by exploiting the intra-signal and inter-signal correlation of the sensor signals. As the number of samples is approximately proportional to the total energy consumed by a $\mathrm{SN}$ in the processes of sampling, processing and transmission, this translates directly into savings in energy consumption and so prolong the life time for a network having battery powered SNs. Experiments demonstrate the advantage of our approach in the reconstruction of typical signals collected by a practical WSN.

\section{REFERENCES}

[1] E. Candes and M. Wakin, "An introduction to compressive sampling," Signal Processing Magazine, IEEE, vol. 25, no. 2, pp. 21 -30, Mar. 2008.

[2] Z. Zhang, Z. Han, H. Li, D. Yang, and C. Pei, "Belief propagation based cooperative compressed spectrum sensing in wideband cognitive radio networks," Wireless Communications, IEEE Transactions on, vol. 10, no. 9 , pp. $3020-3031$, Sept. 2011.

[3] A. Gomaa and N. Al-Dhahir, "A sparsity-aware approach for nbi estimation in mimo-ofdm," Wireless Communications, IEEE Transactions on, vol. 10, no. 6, pp. $1854-1862$, Jun. 2011.

[4] J. Yick, B. Mukherjee, and D. Ghosal, "Wireless sensor network survey," Computer Networks, vol. 52, no. 12, pp. 2292 - 2330, 2008. 
[5] J. Chou, D. Petrovic, and K. Ramachandran, "A distributed and adaptive signal processing approach to reducing energy consumption in sensor networks," in INFOCOM 2003. Twenty-Second Annual Joint Conference of the IEEE Computer and Communications. IEEE Societies, vol. 2, March 2003, pp. 1054-1062.

[6] R. Cristescu, B. Beferull-Lozano, and M. Vetterli, "On network correlated data gathering," in INFOCOM 2004. Twenty-third AnnualJoint Conference of the IEEE Computer and Communications Societies, vol. 4, March 2004, pp. 2571 - 2582 vol.4.

[7] K. Yuen, B. Liang, and L. Baochun, "A distributed framework for correlated data gathering in sensor networks," Vehicular Technology, IEEE Transactions on, vol. 57, no. 1, pp. 578 -593, Jan. 2008

[8] E. Candès and T. Tao, "Decoding by linear programming," IEEE Transactions on Information Theory, vol. 51, no. 12, pp. 4203-4215, 2005.

[9] D. Donoho, "Compressed sensing," IEEE Transactions on Information Theory, vol. 52, no. 4, pp. 1289-1306, 2006.

[10] D. Baron, M. Wakin, M. Duarte, S. Sarvotham, and R. Baraniuk, "Distributed compressed sensing," Technical Report ECE-0612, Electrical and Computer Engineering Department, Rice University, Dec. 2006.

[11] W. Bajwa, J. Haupt, A. Sayeed, and R. Nowak, "Compressive wireless sensing," in Proceedings of the 5th international conference on Information processing in sensor networks. ACM, 2006.

[12] W. Wang, M. Garofalakis, and K. Ramchandran, "Distributed sparse random projections for refinable approximation," in 6th International Symposium on Information Processing in Sensor Networks, IPSN, 2007, pp. 331-339.

[13] N. Vaswani and W. Lu, "Modified-CS: Modifying compressive sensing for problems with partially known support," Signal Processing, IEEE Transactions on, vol. 58, no. 9, pp. 4595-4607, Sept. 2010.

[14] D. Angelosante, J. Bazerque, and G. Giannakis, "Online adaptive estimation of sparse signals: Where RLS meets the $\ell_{1}$-norm," Signal Processing, IEEE Transactions on, vol. 58, no. 7, pp. 3436 -3447, July 2010.

[15] Y. Kopsinis, K. Slavakis, and S. Theodoridis, "Online sparse system identification and signal reconstruction using projections onto weighted $\ell_{1}$ balls," Signal Processing, IEEE Transactions on, vol. 59, no. 3, pp. 936 -952, March 2011

[16] C. Luo, F. Wu, J. Sun, and C. W. Chen, "Efficient measurement generation and pervasive sparsity for compressive data gathering," Wireless Communications, IEEE Transactions on, vol. 9, no. 12, pp. 3728 -3738, Dec. 2010

[17] E. Candes, M. Wakin, and S. Boyd, "Enhancing sparsity by reweighted 1 1 minimization," Journal of Fourier Analysis and Applications, vol. 14 no. 5, pp. 877-905, 2008

[18] P. Bodik, W. Hong, C. Guestrin, S. Madden, M. Paskin, and R. Thibaux. (2004, Feb.) Intel lab data. [Online]. Available: http://db.csail.mit.edu/labdata/labdata.html

[19] E. Candès, "The restricted isometry property and its implications for compressed sensing," Comptes rendus-Mathématique, vol. 346, no. 910, pp. 589-592, 2008.

[20] S. Kim, K. Koh, M. Lustig, S. Boyd, and D. Gorinevsky, "An interiorpoint method for large-scale $\ell_{1}$-regularized least squares," IEEE journal of selected topics in signal processing, vol. 1, no. 4, pp. 606-617, 2007.

[21] M. Figueiredo, R. Nowak, and S. Wright, "Gradient projection for sparse reconstruction: Application to compressed sensing and other inverse problems," IEEE Journal on selected topics in Signal Processing, vol. 1, no. 4, pp. 586-597, 2007.

[22] D. Needell and J. Tropp, "CoSaMP: Iterative signal recovery from incomplete and inaccurate samples," Applied and Computational Harmonic Analysis, vol. 26, no. 3, pp. 301-321, 2009.

[23] W. Dai and O. Milenkovic, "Subspace pursuit for compressive sensing signal reconstruction," Information Theory, IEEE Transactions on, vol. 55, no. 5, pp. 2230-2249, May 2009.

[24] R. Tibshirani, "Regression shrinkage and selection via the lasso," Journal of the Royal Statistical Society Series B Methodological, vol. 58, no. 1, pp. 267-288, 1996.

[25] J. Tropp and A. Gilbert, "Signal recovery from random measurements via orthogonal matching pursuit," IEEE Transactions on Information Theory, vol. 53, no. 12, p. 4655, 2007.

[26] D. Needell and R. Vershynin, "Uniform uncertainty principle and signal recovery via regularized orthogonal matching pursuit," Foundations of computational mathematics, vol. 9, no. 3, pp. 317-334, 2009.

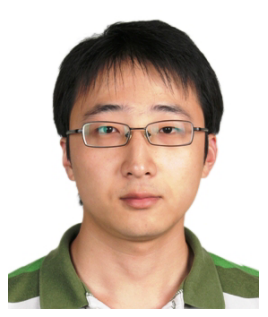

Wei Chen Wei Chen received his B.Eng. degree and M.Eng. degree in Communications Engineering from Beijing University of Posts and Telecommunications, China, in 2006 and 2009, respectively. $\mathrm{He}$ is currently working towards his Ph.D. degree in Computer Science at the Computer Laboratory, University of Cambridge, UK. His current research interests are in compressive sensing, wireless sensor networks, cognitive radio, and image processing.

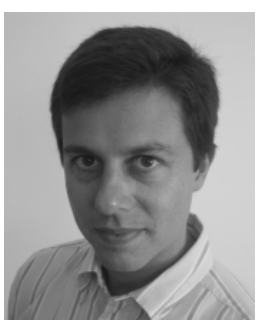

Miguel Rodrigues Miguel Rodrigues (MIEEE) received the Licenciatura degree in Electrical Engineering from the University of Porto, Portugal in 1998 and the Ph.D. degree in Electronic and Electrical Engineering from University College London, U.K. in 2002. He is currently a Senior Lecturer with the Department of Electronic and Electrical Engineering, University College London, UK. He was previously with the Department of Computer Science, University of Porto, Portugal, raising through the ranks from Assistant to Associate Professor, where he also led the Information Theory and Communications Research Group at Instituto de Telecomunicações - Porto. He has held postdoctoral or visiting appointments with various Institutions worldwide including University College London, Cambridge University, Princeton University, and Duke University in the period 2003-2012. His research work, which lies in the general areas of information theory, communications and signal processing, has led to over 100 papers in journals and conferences to date. Dr. Rodrigues was honored by the IEEE ComSoc/IT Society Joint Paper Award in 2011 for his work on Wireless Information-Theoretic Security (joint with M. Bloch, J. Barros and S. M. McLaughlin).

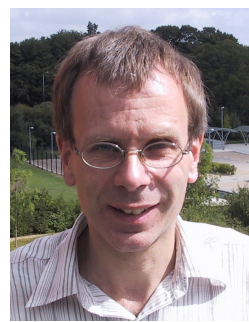

Ian Wassell Ian Wassell received his B.Sc. and B.Eng. degrees from the University of Loughborough in 1983, and his Ph.D. degree from the University of Southampton in 1990 . He is a Senior Lecturer at the University of Cambridge Computer Laboratory and has in excess of 15 years experience in the simulation and design of radio communication systems gained via a number of positions in industry and higher education. He has published more than 170 papers concerning wireless communication systems and his current research interests include: fixed wireless access, sensor networks, cooperative networks, propagation modelling, compressive sensing and cognitive radio. $\mathrm{He}$ is a member of the IET and a Chartered Engineer. 